\title{
Efficient Qualitative Analysis of Classes of Recursive Markov Decision Processes and Simple Stochastic Games
}

\author{
Kousha Etessami ${ }^{1}$ and Mihalis Yannakakis ${ }^{2}$ \\ 1 LFCS, School of Informatics, University of Edinburgh \\ 2 Department of Computer Science, Columbia University
}

\begin{abstract}
Recursive Markov Decision Processes (RMDPs) and Recursive Simple Stochastic Games (RSSGs) are natural models for recursive systems involving both probabilistic and non-probabilistic actions. As shown recently [10], fundamental problems about such models, e.g., termination, are undecidable in general, but decidable for the important class of 1-exit RMDPs and RSSGs. These capture controlled and game versions of multi-type Branching Processes, an important and wellstudied class of stochastic processes. In this paper we provide efficient algorithms for the qualitative termination problem for these models: does the process terminate almost surely when the players use their optimal strategies? Polynomial time algorithms are given for both maximizing and minimizing 1-exit RMDPs (the two cases are not symmetric). For 1-

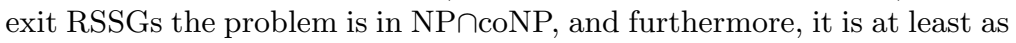
hard as other well-known NP^coNP problems on games, e.g., Condon's quantitative termination problem for finite SSGs ([3]). For the class of linearly-recursive 1-exit RSSGs, we show that the problem can be solved in polynomial time.
\end{abstract}

\section{Introduction}

In recent work [10], we introduced and studied Recursive Markov Decision Processes (RMDPs) and Recursive Simple Stochastic Games (RSSGs), which provide natural models for recursive systems (e.g., programs with procedures) involving both probabilistic and non-probabilistic actions. They define infinitestate MDPs and SSGs that extend Recursive Markov Chains (RMCs) $([8,9])$ with non-probabilistic actions that are controlled by a controller and/or the environment (the "players"). Informally, a recursive model (RMC, RMDP, RSSG) consists of a (finite) collection of finite state component models (resp. MC, MDP, SSG) that can call each other in a potentially recursive manner.

In this paper we focus on the important class of 1-exit RMDPs and 1-exit $R S S G s$, which we will denote by 1-RMDP and 1-RSSG. These are RMDPs and RSSGs where every component contains exactly 1 exit node. Without players, 1RMCs correspond tightly to both Stochastic Context-Free Grammars (SCFGs) and Multi-Type Branching Processes (MT-BPs). Branching processes are an important class of stochastic processes, dating back to the early work of Galton and 
Watson in the 19th century, and continuing in the 20th century in the work of Kolmogorov, Sevastianov, Harris and others for MT-BPs and beyond (see, e.g., [14]). MT-BPs model the growth of a population of objects of distinct types. In each generation each object of a given type gives rise, according to a probability distribution, to a multi-set of objects of distinct types. These stochastic processes have been used in a variety of applications, including in population genetics ([16]), nuclear chain reactions, ([7]), and RNA modeling in computational biology (based on SCFGs) ([22]). SCFGs are also fundamental models in statistical natural language processing (see, e.g., [19]). 1-RMDPs correspond to a controlled version of MT-BPs (and SCFGs): the reproduction of some types can be controlled, while the dynamics of other types is probabilistic as in ordinary MT-BPs; or the controller may be able to influence the reproduction of some types by choosing among a set of probability distributions (e.g., the branching Markov decision chains of [21]). The goal of the controller is either to maximize the probability of extinction or to minimize it (maximize survival probability). This model would also be suitable for analysis of population dynamics under worst-case (or best-case) assumptions for some types and probabilistic assumptions for others. Such controlled MT-BPs can be readily translated to 1-RMDPs, where the types of the MT-BP correspond to the components of the RMDP, extinction in the MT-BP corresponds to termination in the RMDP, and our results can be used for the design of strategies to achieve or prevent extinction.

Among our results in [10], we showed that for maximizing (minimizing) 1RMDPs, the Qualitative Termination Problem (Qual-TP), is in NP (coNP, respectively), and that the same problem for 1-RSSGs is in $\Sigma_{2}^{P} \cap \Pi_{2}^{P}$. Qual-TP is the problem of deciding whether player 1 (the maximizer) has a strategy to force termination with probability 1 , regardless of the strategy employed by player 2 (the minimizer). (In a maximizing 1-RMDP, the only player present is the maximizer, and in a minimizing 1-RMDP the only player present is the minimizer.)

In this paper we improve significantly on the above results. We show that Qual-TP, both for maximizing 1-RMDPs and for minimizing 1-RMDPs, can in fact be decided in polynomial time. It follows easily from this and strong determinacy results from [10], that for 1-RSSGs Qual-TP is in NP^coNP. We show that one can not easily improve on this upper bound, by providing a polynomial time reduction from the Quantitative Termination Problem (Quan-TP) for finite SSGs ([3]) to the Qual-TP problem for 1-RSSGs. Condon [3] showed that for finite SSGs the Quan-TP problem, specifically the problem of deciding whether player

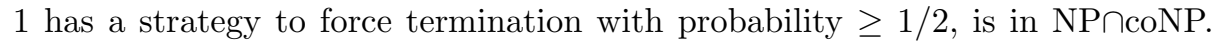
Whether the problem can be solved in $\mathrm{P}$ is a well-known open problem, that includes as special cases several other longstanding problems (e.g., "parity games" and "mean-payoff" games). We note (as is already known) that for finite SSGs, Qual-TP itself is in polynomial time. We in fact show a more general result, namely, that Qual-TP is in polynomial time for the class of 1-RSSGs that are linearly-recursive.

Thus, we provide a new class of infinite-state SSGs whose qualitative decision problem is at least as hard as the quantitative decision problem for finite SSGs, 


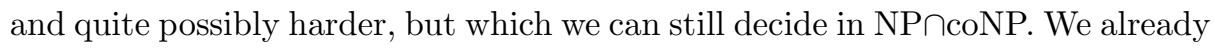
showed in $[10,8]$ that the even harder Quan-TP problem for 1-RSSGs can be decided in PSPACE, and that improving that upper bound even to NP, even for 1-RMCs, would resolve a long standing open problem in the complexity of numerical computation, namely the square-root sum problem ([12]).

Most proofs are omitted due to space.

Related work. Both MDPs and Stochastic Games have a vast literature (see $[20,11]$ ). As mentioned, we introduced RMDPs and RSSGs and studied both quantitative and qualitative termination problems in [10]. We showed that for multi-exit models these problems are undecidable, and that (qualitative) model checking is undecidable even in the 1-exit case. Our earlier work $[8,9]$ developed the theory and algorithms for RMCs and [6,2] studied the related model of probabilistic Pushdown Systems (pPDSs).

Our algorithms here were partly inspired by recent unpublished work by Denardo and Rothblum [4,5] on Multi-Matrix Multiplicative Systems. They study families of square nonnegative matrices, which arise from choosing each matrix row independently from a choice of rows, and they give LP characterizations of when the spectral radius of all matrices in the family will be $\geq 1$ or $>1$. None of our results follow from theirs, but we use techniques similar to theirs, along with other techniques, to obtain our upper bounds.

\section{Definitions and Background}

A Recursive Simple Stochastic Game (RSSG), $A$, is a tuple $A=\left(A_{1}, \ldots, A_{k}\right)$, where each component $A_{i}=\left(N_{i}, B_{i}, Y_{i}, E n_{i}, E x_{i}, \mathrm{pl}_{i}, \delta_{i}\right)$ consists of:

- A set $N_{i}$ of nodes, with a distinguished subset $E n_{i}$ of entry nodes and a (disjoint) subset $E x_{i}$ of exit nodes.

- A set $B_{i}$ of boxes, and a mapping $Y_{i}: B_{i} \mapsto\{1, \ldots, k\}$ that assigns to every box (the index of) a component. To each box $b \in B_{i}$, we associate a set of call ports, Call $b=\left\{(b, e n) \mid e n \in E n_{Y(b)}\right\}$, and a set of return ports, Return $n_{b}=$ $\left\{(b, e x) \mid e x \in E x_{Y(b)}\right\}$. Let Call $^{i}=\cup_{b \in B_{i}}$ Call $_{b}$, Return ${ }^{i}=\cup_{b \in B_{i}}$ Return $_{b}$, and let $Q_{i}=N_{i} \cup$ Call $^{i} \cup$ Return $^{i}$ be the set of all nodes, call ports and return ports; we refer to these as the vertices of component $A_{i}$.

- A mapping $\mathrm{pl}_{i}: Q_{i} \mapsto\{0,1,2\}$ that assigns to every vertex a player (Player 0 represents "chance" or "nature"). We assume $\mathrm{pl}_{i}(e x)=0$ for all $e x \in E x_{i}$.

- A transition relation $\delta_{i} \subseteq\left(Q_{i} \times(\mathbb{R} \cup\{\perp\}) \times Q_{i}\right)$, where for each tuple $(u, x, v) \in \delta_{i}$, the source $u \in\left(N_{i} \backslash E x_{i}\right) \cup \operatorname{Return}^{i}$, the destination $v \in$ $\left(N_{i} \backslash E n_{i}\right) \cup$ Call $^{i}$, and $x$ is either (i) a real number $p_{u, v} \in(0,1]$ (the transition probability) if $\mathrm{pl}_{i}(u)=0$, or (ii) $x=\perp$ if $\mathrm{pl}_{i}(u)=1$ or 2 . For computational purposes we assume that the given probabilities $p_{u, v}$ are rational. Furthermore they must satisfy the consistency property: for every $u \in \mathrm{pl}_{i}^{-1}(0), \sum_{\left\{v^{\prime} \mid\left(u, p_{u, v^{\prime}}, v^{\prime}\right) \in \delta_{i}\right\}} p_{u, v^{\prime}}=1$, unless $u$ is a call port or exit node, neither of which have outgoing transitions, in which case by default $\sum_{v^{\prime}} p_{u, v^{\prime}}=0$. 
We use the symbols $(N, B, Q, \delta$, etc.) without a subscript, to denote the union over all components. Thus, e.g., $N=\cup_{i=1}^{k} N_{i}$ is the set of all nodes of $A$, $\delta=\cup_{i=1}^{k} \delta_{i}$ the set of all transitions, etc.

An RSSG $A$ defines a global denumerable Simple Stochastic Game (SSG) $M_{A}=\left(V=V_{0} \cup V_{1} \cup V_{2}, \Delta, \mathrm{pl}\right)$ as follows. The global states $V \subseteq B^{*} \times Q$ of $M_{A}$ are pairs of the form $\langle\beta, u\rangle$, where $\beta \in B^{*}$ is a (possibly empty) sequence of boxes and $u \in Q$ is a vertex of $A$. More precisely, the states $V \subseteq B^{*} \times Q$ and transitions $\Delta$ are defined inductively as follows:

1. $\langle\epsilon, u\rangle \in V$, for $u \in Q$. ( $\epsilon$ denotes the empty string.)

2. if $\langle\beta, u\rangle \in V \&(u, x, v) \in \delta$, then $\langle\beta, v\rangle \in V$ and $(\langle\beta, u\rangle, x,\langle\beta, v\rangle) \in \Delta$.

3. if $\langle\beta,(b, e n)\rangle \in V \&(b, e n) \in C_{\text {Call }}$, then $\langle\beta b, e n\rangle \in V \&(\langle\beta,(b, e n)\rangle, 1,\langle\beta b, e n\rangle) \in \Delta$.

4. if $\langle\beta b, e x\rangle \in V \&(b, e x) \in \operatorname{Return}_{b}$, then $\langle\beta,(b, e x)\rangle \in V \&(\langle\beta b, e x\rangle, 1,\langle\beta,(b, e x)\rangle) \in \Delta$.

The mapping pl : $V \mapsto\{0,1,2\}$ is given as follows: $\mathrm{pl}(\langle\beta, u\rangle)=\mathrm{pl}(u)$ if $u$ is in $Q \backslash($ Call $\cup E x)$, and $\mathrm{pl}(\langle\beta, u\rangle)=0$ if $u \in$ Call $\cup E x$. The set of vertices $V$ is partitioned into $V_{0}, V_{1}$, and $V_{2}$, where $V_{i}=\mathrm{pl}^{-1}(i)$. We consider $M_{A}$ with various initial states of the form $\langle\epsilon, u\rangle$, denoting this by $M_{A}^{u}$. Some states of $M_{A}$ are terminating states and have no outgoing transitions. These are states $\langle\epsilon, e x\rangle$, where $e x$ is an exit node.

An RSSG where $V_{2}=\emptyset\left(V_{1}=\emptyset\right)$ is called a maximizing (minimizing, respectively) Recursive Markov Decision Process (RMDP); an RSSG where $V_{1} \cup V_{2}=\emptyset$ is called a Recursive Markov Chain (RMC) $([8,9])$; an RSSG where $V_{0} \cup V_{2}=\emptyset$ is called a Recursive Graph or Recursive State Machine(RSM) ([1]). Define 1$R S S G s$ to be those RSSGs where every component has 1 exit, and likewise define 1-RMDPs and 1-RMCs. W.l.o.g., we can assume every component has 1 entry, because multi-entry RSSGs can be transformed to equivalent 1-entry RSSGs with polynomial blowup (similar to RSM transformations [1]). This is not so for exits, e.g., qualitative termination is undecidable for multi-exit RMDPs, whereas it is decidable for 1-RSSGs (see [10]). This entire paper is focused on 1-RSSGs and 1-RMDPs. Accordingly, some of our notation is simpler than that used for general RSSGs in [10]. We shall call a 1-RSSG (1-RMDP, etc.) linear if there in no path of transitions in any component from any return port to a call port.

Our basic goal is to answer qualitative termination questions for 1-RSSGs: "Does player 1 have a strategy to force the game to terminate at exit ex (i.e., reach $\langle\epsilon, e x\rangle)$, starting at $\langle\epsilon, u\rangle$, with probability 1 , regardless of how player 2 plays?". A strategy $\sigma$ for player $i, i \in\{1,2\}$, is a function $\sigma: V^{*} V_{i} \mapsto V$, where, given the history $w s \in V^{*} V_{i}$ of play so far, with $s \in V_{i}$ (i.e., it is player $i$ 's turn to play a move), $\sigma(w s)=s^{\prime}$ determines the next move of player $i$, where $\left(s, \perp, s^{\prime}\right) \in \Delta$. (We could also allow randomized strategies.) Let $\Psi_{i}$ denote the set of all strategies for player $i$. A pair of strategies $\sigma \in \Psi_{1}$ and $\tau \in \Psi_{2}$ induce in a straightforward way a Markov chain $M_{A}^{\sigma, \tau}=\left(V^{*}, \Delta^{\prime}\right)$, whose set of states is the set $V^{*}$ of histories. Given an initial vertex $u$, suppose $e x$ is the unique exit node of $u$ 's component. Let $q_{u}^{*, \sigma, \tau}$ be the probability that, in $M_{A}^{\sigma, \tau}$, starting at initial state $\langle\epsilon, u\rangle$ we will eventually terminate, by reaching some $w\langle\epsilon, e x\rangle$, for $w \in V^{*}$. From general determinacy results (e.g., [18]) it follows that $\sup _{\sigma \in \Psi_{1}} \inf _{\tau \in \Psi_{2}} q_{u}^{*, \sigma, \tau}=\inf _{\tau \in \Psi_{2}} \sup _{\sigma \in \Psi_{1}} q_{u}^{*, \sigma, \tau}$. This is the value of the game 
starting at $u$, which we denote by $q_{u}^{*}$. We are interested in the following problem: Qual-TP: Given $A$, a 1-RSSG (or 1-RMDP), and given a vertex $u$ in $A$, is $q_{u}^{*}=1$ ?

For a strategy $\sigma \in \Psi_{1}$, let $q_{u}^{*, \sigma}=\inf _{\tau \in \Psi_{2}} q_{u}^{*, \sigma, \tau}$, and for $\tau \in \Psi_{2}$, let $q_{u}^{*, \cdot, \tau}=$ $\sup _{\sigma \in \Psi_{1}} q_{u}^{*, \sigma, \tau}$. We showed in [10] that 1-RSSGs satisfy a strong form of memoryless determinacy, namely, call a strategy Stackless $\&$ Memoryless (SEM) if it depends neither on the history of the game nor on the current call stack, i.e., only depends on the current vertex. We call a game $S \mathscr{E} M M$-determined if both players have S\&M optimal strategies.

Theorem 1. ([10]) Every 1-RSSG termination game is SEBM-determined. (Moreover, there is an $S \mathscr{B} M$ strategy $\sigma^{*} \in \Psi_{1}$ that maximizes the value of $q_{u}^{*, \sigma}$ for all $u$, and likewise $a \tau^{*} \in \Psi_{2}$ that minimizes the value of $q_{u}^{*, \cdot, \tau}$ for all u.)

For multi-exit RMDPs and RSSGs things are very different. We showed that even memoryless determinacy fails badly (there may not exist any optimal strategy at all, only $\epsilon$-optimal ones)), and furthermore Qual-TP is undecidable (see [10]).

Note that there are finitely many S\&M strategies for player $i$ : each picks one edge out of each vertex belonging to player $i$. For 1-RMCs, where there are only probabilistic vertices, we showed in [8] that Qual-TP can be decided in polynomial time, using a spectral radius characterization for certain moment matrices associated with 1-RMCs. It followed, by guessing strategies, that Qual-TP for both maximizing and minimizing 1-RMDPs is in NP, and that Qual-TP for 1RSSGs is in $\Sigma_{2}^{P} \cap \Pi_{2}^{P}$. We obtain far stronger upper bounds in this paper. We will also use the following fact from [10].

Proposition 1. ([10]) We can decide in P-time if the value of a 1-RSSG termination game (and optimal termination probability in a maximizing or minimizing 1-RMDP) is exactly 0.

In ([10]) we defined a monotone system $S_{A}$ of nonlinear min-max equations for 1-RSSGs $A$, and showed that its Least Fixed Point solution yields the desired probabilities $q_{u}^{*}$. These systems generalize both the linear Bellman's equations for MDPs, as well as the nonlinear system of polynomial equation for RMCs studied in [8]. Here we recall these systems of equations (with a slightly simpler notation). Let us use a variable $x_{u}$ for each unknown $q_{u}^{*}$, and let $\mathbf{x}$ be the vector of all $x_{u}, u \in Q$. The system $S_{A}$ has one equation of the form $x_{u}=P(\mathbf{x})$ for each vertex $u$. Suppose that $u$ is in component $A_{i}$ with (unique) exit ex. There are 5 cases based on the "Type" of $u$.

1. $u \in$ Type $_{1}: u=e x$. In this case: $x_{u}=1$.

2. $u \in$ Type $_{\text {rand }}: \mathrm{pl}(u)=0 \& u \in\left(N_{i} \backslash\{e x\}\right) \cup \operatorname{Return}^{i}: x_{u}=\sum_{\left\{v \mid\left(u, p_{u, v}, v\right) \in \delta\right\}} p_{u, v} x_{v}$.

(If $u$ has no outgoing transitions, this equation is by definition $x_{u}=0$.)

3. $u \in$ Type $_{\text {call }}: u=(b, e n)$ is a call port: $x_{(b, e n)}=x_{e n} \cdot x_{\left(b, e x^{\prime}\right)}$, where $e x^{\prime} \in$ $E x_{Y(b)}$ is the unique exit of $A_{Y(b)}$.

4. $u \in$ Type $_{\text {max }}: \mathrm{pl}(u)=1 \& u \in\left(N_{i} \backslash\{\right.$ ex $\left.\}\right) \cup$ Return $^{i}: x_{u}=\max _{\{v \mid(u, \perp, v) \in \delta\}} x_{v}$.

(If $u$ has no outgoing transitions, we define $\max (\emptyset)=0$.)

5. $u \in$ Type $_{\text {min }}: \operatorname{pl}(u)=2$ and $u \in\left(N_{i} \backslash\{\right.$ ex $\left.\}\right) \cup$ Return $^{i}: x_{u}=\min _{\{v \mid(u, \perp, v) \in \delta\}} x_{v}$. (If $u$ has no outgoing transitions, we define $\min (\emptyset)=0$.) 
In vector notation, we denote the system $S_{A}$ by $\mathbf{x}=P(\mathbf{x})$.

Given 1-RSSG $A$, we can easily construct $S_{A}$ in linear time. For vectors $\mathbf{x}, \mathbf{y} \in \mathbb{R}^{n}$, define $\mathbf{x} \leq \mathbf{y}$ to mean $x_{j} \leq y_{j}$ for every coordinate $j$. Let $\mathbf{q}^{*} \in \mathbb{R}^{n}$ denote the $n$-vector of $q_{u}^{*}$ 's.

Theorem 2. ([10]) Let $\mathbf{x}=P(\mathbf{x})$ be the system $S_{A}$ associated with 1-RSSG A. Then $\mathbf{q}^{*}=P\left(\mathbf{q}^{*}\right)$, and for all $\mathbf{q}^{\prime} \in \mathbb{R}_{\geq 0}^{n}$, if $\mathbf{q}^{\prime}=P\left(\mathbf{q}^{\prime}\right)$, then $\mathbf{q}^{*} \leq \mathbf{q}^{\prime}$ (in other words, $\mathbf{q}^{*}$ is the Least Fixed Point, of $P: \mathbb{R}_{\geq 0}^{n} \mapsto \mathbb{R}_{\geq 0}^{n}$ ).

\section{Qualitative termination for 1-RMDPs in P-time}

We show that, for both maximizing 1-RMDPs and minimizing 1-RMDPs, qualitative termination can be decided in polynomial time. Please note: the two cases are not symmetric. We provide distinct algorithms for each of them. An important result for us is this:

Theorem 3. ([8]) Qual-TP for 1-RMCs is decidable in polynomial time.

We briefly indicate the key elements of that upper bound (please see [8] for more details). Our algorithm employed a spectral radius characterization of moment matrices associated with 1-RMCs. Given the system of polynomial equations $x=P(x)$ for a 1-RMC (no min and max types), its moment matrix $B$ is the square Jacobian matrix of $P(x)$, whose $(i, j)$ 'th entry is the partial derivative $\partial P_{i}(x) / \partial x_{j}$, evaluated at the all 1 vector (i.e., $x_{u} \leftarrow 1$ for $u \in Q$ ). We showed in [8] that if the system $x=P(x)$ is decomposed into strongly connected components (SCCs) in a natural way, and we associate a moment matrix $B_{C}$ to each SCC, $C$, then $q_{u}^{*}=1$ for every $u$ where $x_{u}$ is in $C$, iff either $u$ is of Type ${ }_{1}$, or [C has successor SCCs and $q_{v}^{*}=1$ for all nodes $v$ in any successor SCC of $C$, and $\rho\left(B_{C}\right) \leq 1$, where $\rho(M)$ is the spectral radius of a square matrix $\left.M\right]$.

Theorem 4. Given a maximizing 1-RMDP, A, and a vertex $u$ of $A$, we can decide in polynomial time whether $q_{u}^{*}=1$. In other words, for maximizing 1$R M D P s$, Qual-TP is in $\mathbf{P}$.

Proof. Given a maximizing 1-RMDP, $A$, we shall determine for all vertices $u$, whether $q_{u}^{*}=1, q_{u}^{*}=0$, or $0<q_{u}^{*}<1$. The system of equations $x=P(x)$ for $A$ defines a labeled dependency graph, $G_{A}=(Q, \rightarrow)$, as follows: the nodes $Q$ of $G_{A}$ are the vertices of $A$, and there is an edge $u \rightarrow v$ iff $x_{v}$ appears on the right hand side of the equation $x_{u}=P_{u}(x)$. Each node $u$ is labeled by its Type. If $u \in$ Type $_{\text {rand }}$, i.e., $u$ is a probabilistic vertex, and $x_{v}$ appears in the weighted sum $P_{u}(x)$ as a term $p_{u, v} x_{v}$, then the edge from $u$ to $v$ is labeled by the probability $p_{u, v}$. Otherwise, the edge is unlabeled.

We wish to partition the nodes of the dependency graph into three classes: $Z_{0}=\left\{u \mid q_{u}^{*}=0\right\}, Z_{1}=\left\{u \mid q_{u}^{*}=1\right\}$, and $Z_{\$}=\left\{u \mid 0<q_{u}^{*}<1\right\}$. In our algorithm we will use a fourth partition, $Z_{\text {? }}$, to denote those nodes for which we have not yet determined to which partition they belong. We first compute $Z_{0}$. By proposition 1, this can be done easily in P-time even for 1-RSSGs. Once 
we have computed $Z_{0}$, the remaining nodes belong either to $Z_{1}$ or $Z_{\$}$. Clearly, Type ${ }_{1}$ nodes belong to $Z_{1}$.

Initialize: $Z_{1} \leftarrow$ Type ${ }_{1} ; Z_{\$} \leftarrow \emptyset$; and $Z_{\text {? }} \leftarrow Q \backslash\left(Z_{1} \cup Z_{0}\right)$;

Next, we do one "preprocessing" step to categorize some remaining "easy" nodes into $Z_{1}$ and $Z_{\$}$, as follows:

repeat

if $u \in Z_{\text {? }} \cap\left(\right.$ Type $_{\text {rand }} \cup$ Type call $)$ has all of its successors in $Z_{1}$

then $Z_{?} \leftarrow Z_{\text {? }} \backslash\{u\} ; Z_{1} \leftarrow Z_{1} \cup\{u\}$;

if $u \in Z_{\text {? }} \cap$ Type $_{\max }$ has some successor in $Z_{1}$

then $Z_{\text {? }} \leftarrow Z_{\text {? }} \backslash\{u\} ; Z_{1} \leftarrow Z_{1} \cup\{u\}$;

if $u \in Z_{\text {? }} \cap\left(\right.$ Type $_{\text {rand }} \cup$ Type $\left._{\text {call }}\right)$ has some successor in $Z_{0} \cup Z_{\$}$

then $Z_{\text {? }} \leftarrow Z_{\text {? }} \backslash\{u\} ; Z_{\$} \leftarrow Z_{\$} \cup\{u\}$;

if $u \in Z_{\text {? }} \cap$ Type max $_{\text {mas }}$ all successors in $\left(Z_{0} \cup Z_{\S}\right)$

then $Z_{\text {? }} \leftarrow Z_{\text {? }} \backslash\{u\} ; Z_{\$} \leftarrow Z_{\$} \cup\{u\}$;

until (there is no change to $Z_{\text {? }}$ )

The preprocessing step will not, in general, empty $Z_{\text {? }}$, and we need to categorize the remaining nodes in $Z_{\text {? }}$. We will construct a set of linear inequalities (an LP without an objective function) which has a solution iff there are any remaining node in $Z_{\text {? }}$ which belongs in $Z_{1}$, and if so, the solution we obtain to

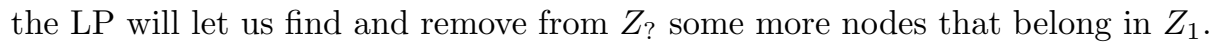

Note that, if we can do this, then we can solve our problem, because all we need to do is iterate: we repeatedly do a preprocessing step, followed by the LP step to remove nodes from $Z_{\text {? }}$, until no more nodes can be removed, at which point we are done: the remaining nodes in $Z_{\text {? }}$ all belong to $Z_{\$}$.

For the LP step, restrict attention to the vertices remaining in $Z$ ? . These vertices induce a subgraph of $G_{A}$, call it $G_{A}^{\prime}$. Call a remaining probabilistic

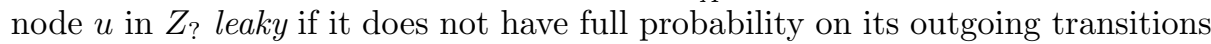
inside $G_{A}^{\prime}$. Note that this happens if and only if some of $u$ 's outedges in $G_{A}$ lead to nodes in $Z_{1}$ (otherwise, if $u$ had an outedge to a node in $Z_{0}$ or $Z_{\$}$, it would already have been removed from $Z$ ? during preprocessing). Let $\mathcal{L}$ denote the set of remaining leaky nodes in $Z_{\text {? }}$. We add an extra terminal node $t$ to $G_{A}^{\prime}$, and for every $u \in \mathcal{L}$ we add a probabilistic edge $u \stackrel{p_{u, t}}{\rightarrow} t$, where $p_{u, t}=1-\sum_{v \in Z_{?}} p_{u, v}$.

W.l.o.g., assume that both entries of components and return nodes are probabilistic nodes (this can easily be assured by minor adjustments to the input 1-RSSG). The LP has a variable $y_{i}$ for every node $i \in Z_{\text {? }}$ that is not Type $e_{\max }$, and has a variables $y_{i, j}$ for every Type $e_{\max }$ node $i \in Z_{\text {? }}$ and successor $j \in Z_{\text {? }}$ of $i$. In addition there are flow variables $f_{i, j, k}$ for each node $i \in Z_{\text {? }}$, and every edge $j \rightarrow k$ in $G_{A}^{\prime}$. The constraints are as follows.

1. For every $j \in$ Type $_{\text {rand }} \cup$ Type $_{\text {call }}$ that is not a component entry or a return:

$$
y_{j} \geq \sum_{i \rightarrow j \wedge} p_{i \in \text { Type }_{\text {rand }}} y_{i}+\sum_{i \rightarrow j \wedge} y_{i \in \text { Type }_{\max }} y_{i, j}
$$

2. For every $j \in$ Type $_{\max }$ :

$$
\sum_{k} y_{j, k} \geq \sum_{i \rightarrow j \wedge i \in \text { Type }_{\text {rand }}} p_{i, j} y_{i}+\sum_{i \rightarrow j \wedge i \in \text { Type }_{\max }} y_{i, j}
$$


3. For every node $i$ that is the entry of a component, say $A_{r}$ :

$$
y_{i} \geq \sum_{j=(b, e n) \in \text { Type }_{\text {call }} \wedge Y(b)=r} y_{j}
$$

4. For every node $i$ that is a return node, say of box $b: y_{i} \geq y_{j}$, where $j$ is the call node of $b$.

5. $\sum_{i} y_{i}+\sum_{i, j} y_{i, j}=1$.

6. $y \geq 0$.

Regard the dependency graph as a network flow graph with capacity on each edge $i \rightarrow j$ coming out of a max node equal to $y_{i, j}$ and capacity of edges $i \rightarrow j$ coming out of the other vertices equal to $y_{i}$. We set up one flow problem for each $i \in Z_{\text {? }}$, with source $i$, sink $t$ and flow variables $f_{i j k}$.

7. For every vertex $i$, we have flow conservation constraints on the variables $f_{i, j, k}$, i.e., $\sum_{k} f_{i, j, k}=\sum_{k} f_{i, k, j}$, for all nodes $j \in Z_{\text {? }}, j \neq i, t$.

8. Nonnegativity constraints: $f_{i, j, k} \geq 0$ for all $i, j, k$.

9. Capacity constraints: $f_{i, j, k} \leq y_{j, k}$ for every $j \in$ Type $_{\max }$ with successor $k$, and for every node $i$; and $f_{i, j, k} \leq y_{j}$ for every $j \in$ Type $_{\text {rand }} \cup$ Type $_{\text {call }}$ and successor $k$ in $G_{A}^{\prime}$ and every node $i$.

10. Source constraints: $\sum_{k} f_{i, i, k}=y_{i} / 2^{2 m}$, for every $i \in$ Type $_{\text {rand }} \cup$ Type $_{\text {call }}$, and $\sum_{k} f_{i, i, k}=\sum_{j} y_{i, j} / 2^{2 m}$, for $i \in$ Type $_{\max }$, where $m$ is defined as follows. Suppose our LP in constraints (1.-6.) has $r$ variables and constraints, and that its rational entries have numerator and denominator with at most $l$ bits. If there is a solution to (1.-6.), then (see, e.g., [13]), there is a rational solution whose numerators and denominators require at most $m=\operatorname{poly}(r, l)$ bits to encode, where poly $(r, l)$ is a polynomial in $r$ and $l$. Note $r \in O\left(\left|G_{A}^{\prime}\right|\right)$, $l$ is bounded by the number of bits required for the transition probabilities $p_{u, v}$ in $A$, hence $m$ is polynomial in the input size.

The purpose of constraints (7-10) is to ensure that every vertex with a nonzero $y$ variable can reach a leaky vertex in the subgraph of $G_{A}^{\prime}$ induced by the support of the $y$ solution vector.

Lemma 1. There exists a vertex $u \in Z_{\text {? }}$ such that $q_{u}^{*}=1$ if and only if the $L P$ constraints in (1.-10.) are feasible. Moreover, from a solution to the LP we can find a (partial) strategy for the maximizing player that forces termination from some such $u$ with probability $=1$.

So to summarize, we set up and solve the LP. If there is no solution, then for all remaining vertices $u \in Z_{?}, q_{u}^{*}<1$, and thus $u \in Z_{\$}$. If there is a solution, use the above partial (randomized) strategy for some of the max nodes, leaving the strategy for other nodes unspecified. This allows us to set to 1 some vertices (vertices in the bottom SCC's of the resulting 1-RMC), and thus to move them to $Z_{1}$. We can then iterate the preprocessing step and then the LP step until we reach a fixed point, at which point we have categorized all vertices $u$ into one of $Z_{0}, Z_{1}$ or $Z_{\$}$. 
Theorem 5. Given a minimizing 1-RMDP, A, and a vertex $u$ of $A$, we can decide in polynomial time whether $q_{u}^{*}=1$. In other words, for minimizing 1RMDPs, Qual-TP is in $\mathbf{P}$.

Proof. As in the previous theorem, we want to classify the vertices into $Z_{0}, Z_{\$}, Z_{1}$, this time under optimal play of the minimizing player. We again consider the dependency graph $G_{A}$ of $A$. We will again use $Z$ ? to denote those vertices that have not yet been classified.

Initialize: $Z_{1} \leftarrow$ Type ${ }_{1} ; Z_{\$} \leftarrow \emptyset$; and $Z_{\text {? }} \leftarrow Q \backslash\left(Z_{1} \cup Z_{0}\right)$;

Next, we again do a "preprocessing" step, which is "dual" to that of the preprocessing we did for maximizing 1-RMDPs, and categorizes some remaining "easy" nodes into $Z_{1}$ and $Z_{\$}$ :

repeat

if $u \in Z_{\text {? }} \cap\left(\right.$ Type $_{\text {rand }} \cup$ Type $\left._{\text {call }}\right)$ has all of its successors in $Z_{1}$

then $Z_{\text {? }} \leftarrow Z_{\text {? }} \backslash\{u\} ; Z_{1} \leftarrow Z_{1} \cup\{u\}$;

if $u \in Z_{\text {? }} \cap$ Type $_{\min }$ has some successor in $Z_{\$}$

then $Z_{\text {? }} \leftarrow Z_{\text {? }} \backslash\{u\} ; Z_{\$} \leftarrow Z_{\$} \cup\{u\}$;

if $u \in Z_{\text {? }} \cap\left(\right.$ Type $_{\text {rand }} \cup$ Type $\left._{\text {call }}\right)$ has some successor in $Z_{0} \cup Z_{\$}$

then $Z_{\text {? }} \leftarrow Z_{\text {? }} \backslash\{u\} ; Z_{\$} \leftarrow Z_{\$} \cup\{u\}$;

if $u \in Z_{\text {? }} \cap$ Type $_{\text {min }}$ has all successors in $\left(Z_{1}\right)$

then $Z_{\text {? }} \leftarrow Z_{\text {? }} \backslash\{u\} ; Z_{1} \leftarrow Z_{1} \cup\{u\}$

until (there is no change to $Z_{\text {? }}$ )

Note that, after the preprocessing step, for every edge $u \rightarrow v$ in $G_{A}$ from $u \in Z_{\text {? }}$ to $v \notin Z_{\text {? }}$, it must be the case that $v \in Z_{1}$ (otherwise, $u$ would have already been moved to $Z_{\$}$ or $Z_{0}$ ). After preprocessing, we formulate a (different) LP, which has a solution iff there are more nodes currently in $Z_{\text {? }}$ which belong in $Z_{\$}$. Restrict attention to nodes in $Z_{\text {? }}$, and consider the subgraph $G_{A}^{\prime}$ of $G_{A}$ induced by the nodes in $Z_{\text {? }}$. The LP has a variable $y_{i}$ for every remaining vertex $i \in Z_{\text {? }}$ such that $i \notin$ Type $_{\min }$, and has a variable $y_{i j}$ for every (remaining) node $i \in$ Type $_{\text {min }}$, and successor $j$ of $i$ in $G_{A}^{\prime}$. We shall need the following lemma:

Lemma 2. Consider a square nonnegative matrices $B$ with at most $n$ rows and having rational entries with at most $l$ bits each. If $\rho(B)>1$ then $\rho(B) \geq 1+1 / 2^{m}$ where $m=\operatorname{poly}(n, l)$ and $\operatorname{poly}(n, l)$ is some polynomial in $n$ and $l$.

Let $d=\left(1+1 / 2^{m}\right)$. The constraints of our LP are as follows. For the LP we restrict attention to only those nodes $j, i$ in $Z_{\text {? }}$.

1. For every $j \in$ Type $_{\text {rand }}$ that is not a component entry or a return, as well as for every $j \in$ Type $_{\text {call }}$ :

$$
d y_{j} \leq \sum_{i \in \text { Type }_{\text {rand }} \wedge i \rightarrow j} p_{i, j} y_{i}+\sum_{i \in \text { Type }_{\text {min }} \wedge i \rightarrow j} y_{i, j}
$$

2. For every $j \in$ Type $_{\min }$ :

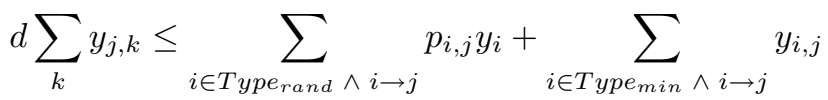


3. For every node $i$ that is the entry of a component, say $A_{r}$ :

$$
d y_{i} \leq \sum_{j=(b, e n) \in \text { Type }_{\text {call }} \wedge Y(b)=r} y_{j}
$$

4. For every node $i$ that is a return node, say of box $b: d y_{i} \leq y_{j}$, where $j$ is the entry node of $b$.

5. $\sum_{i} y_{i}+\sum_{i, j} y_{i, j}=1$.

6. $y \geq 0$.

Lemma 3. There exists a vertex $u \in Z_{\text {? }}$ such that $q_{u}^{*}<1$ if and only if the LP in (1. - 6.) is feasible. Moreover, from a solution to the LP we can find a (partial) strategy that forces termination from some such $u$ with probability $<1$.

To summarize, we find $Z_{0}$, then do preprocessing to determines the "easy" $Z_{1}$ and $Z_{\$}$ nodes. Then, we set up and solve the LP, finding some more $Z_{\$}$ vertices, removing them, and iterating again with a preprocessing and LP step, until we exhaust $Z_{\text {? }}$ or there is no solution to the LP; in the latter case the remaining vertices all belong to $Z_{1}$. As for a strategy that achieves these assignments, in each iteration when we solve the LP we fix the strategy for certain of the min nodes in a way that ensures that some new vertices will be added to $Z_{\$}$ and leave the other min nodes undetermined. Moreover, in preprocessing, if Type min $_{\text {in }}$ nodes get assigned $Z_{\$}$ based on an outedge, we fix the strategy at that node accordingly.

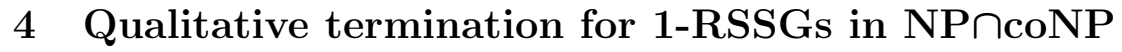

The following is a simple corollary of Theorems 1, 4, and 5 .

Corollary 1. Given a 1-RSSG, A, and given a vertex $u$ of $A$, we can decide in both $N P$ and coNP whether $q_{u}^{*}=1$. In other words, the qualitative termination

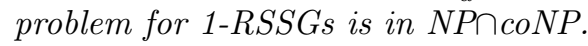

As the following theorem shows, it will not be easy to improve this upper bound. Note that finite SSGs, defined by Condon [3], are a special case of 1RSSGs (we can simply identify the terminal node "1" of the SSG with the unique exit of a single component with no boxes). Define the quantitative termination problem for finite SSGs to be the problem of deciding, given a finite SSG $G$, and a vertex $u$ of $G$, whether $q_{u}^{*} \geq 1 / 2$. Condon [3] showed that this problem is in $\mathrm{NP} \cap \mathrm{coNP}$, and it has been a major open problem whether this upper bound can be improved to P-time.

Theorem 6. There is a P-time reduction from the quantitative termination problem for finite SSGs to the qualitative termination problem for 1-RSSGs.

It is not at all clear whether there is a reduction from qualitative termination for 1-RSSGs to quantitative termination for finite SSGs. Thus, Qual-TP for 1-

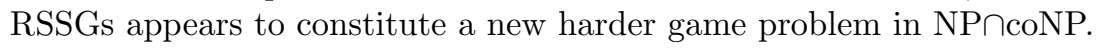




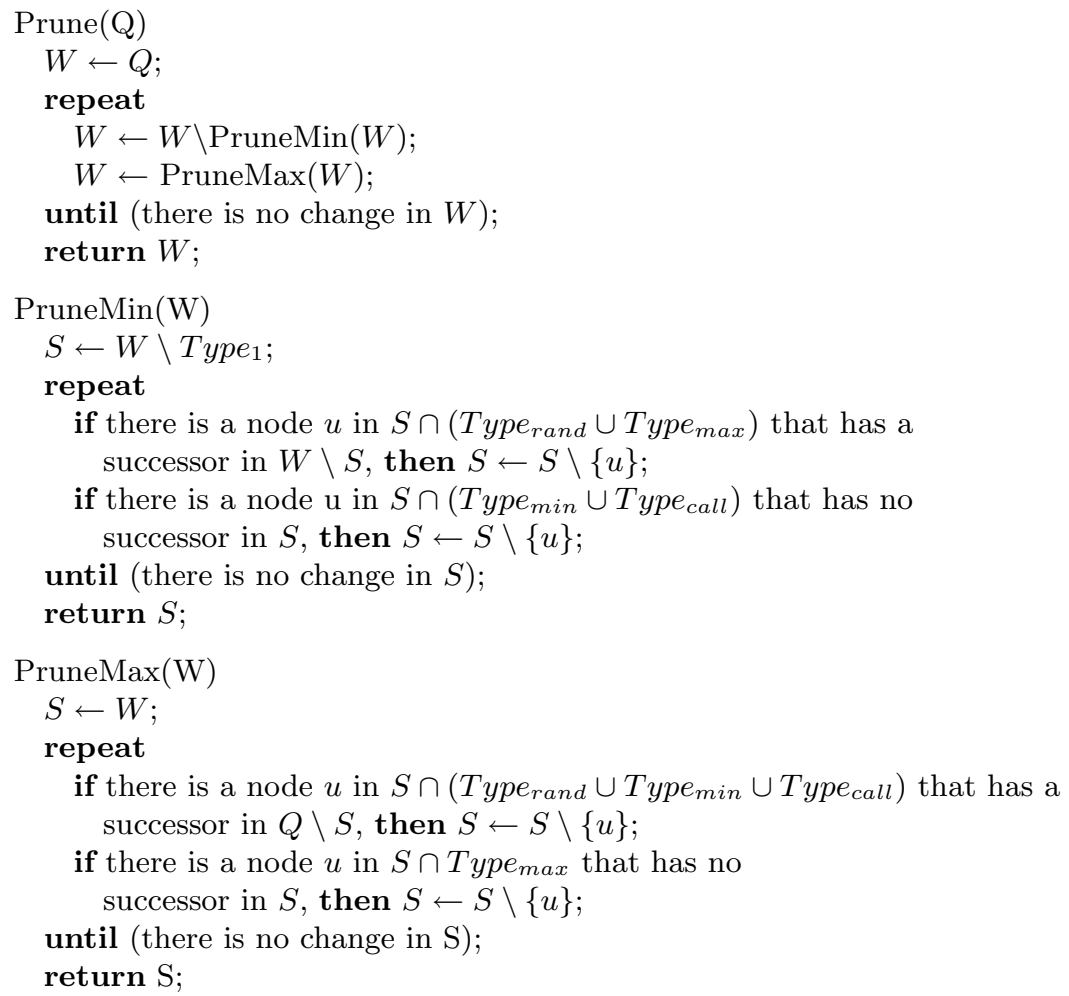

Fig. 1. P-time qualitative termination algorithm for linear 1-RSSGs

\section{Qualitative termination for linear 1-RSSGs in P-time}

We now show that for linear 1-RSSGs, there is a P-time algorithm for deciding Qual-TP. This generalizes of course the case of flat games.

Theorem 7. Given a linear 1-RSSG, and a vertex u, there is a polynomial time algorithm to decide whether $q_{u}^{*}=1$.

Proof. Given a linear 1-RSSG, $A$, consider its dependency graph $G_{A}$. The nodes of partitioned partitioned into 5 types: Type $\max _{\text {max }}$, Type $_{\min }$, Type $_{\text {rand }}$, Type call $_{\text {, }}$, and Type ${ }_{1}$. Let $Q$ be the set of all vertices of $G_{A}$. Our algorithm is depicted in Figure 1. We claim that a call to $\operatorname{Prune}(Q)$ returns precisely those vertices in $Z_{1}=\left\{u \mid q_{u}^{*}=1\right\}$. The proof is omitted due to space.

The algorithm applies more generally to piecewise linear 1-RSSGs, where every vertex $v \in$ Type $_{\text {call }}$ has at most one successor in the dependency graph $G_{A}$ that is in the same SCC as $v$.

Acknowledgment: Thanks to Uri Rothblum for providing us a copy of his unpublished work $[4,5]$. Research partially supported by NSF Grant CCF-0430946 . 


\section{References}

1. R. Alur, M. Benedikt, K. Etessami, P. Godefroid, T. W. Reps, and M. Yannakakis. Analysis of recursive state machines. In ACM Trans. Progr. Lang. Sys., 27:786-818, 2005.

2. T. Brázdil, A. Kučera, and O. Stražovský. Decidability of temporal properties of probabilistic pushdown automata. In Proc. of STACS'05, 2005.

3. A. Condon. The complexity of stochastic games. Inf. E Comp., 96(2):203-224, 1992.

4. E. V. Denardo and U. G. Rothblum. Totally expanding multiplicative systems. unpublished manuscript, submitted, 2005.

5. E. V. Denardo and U. G. Rothblum. A turnpike theorem for a risk-sensitive Markov decision process with stopping. unpublished manuscript, submitted, 2005.

6. J. Esparza, A. Kučera, and R. Mayr. Model checking probabilistic pushdown automata. In Proc. of 19th IEEE LICS'04, 2004.

7. C. J. Everett and S. Ulam. Multiplicative systems, part i., ii, and iii. Technical Report 683,690,707, Los Alamos Scientific Laboratory, 1948.

8. K. Etessami and M. Yannakakis. Recursive Markov chains, stochastic grammars, and monotone systems of nonlinear equations. In Proc. of 22nd STACS'05. Springer, vol. 3404 of LNCS , 2005.

9. K. Etessami and M. Yannakakis. Algorithmic verification of recursive probabilistic state machines. In Proc. 11th TACAS'05, Springer, vol. 3440 of LNCS, 2005.

10. K. Etessami and M. Yannakakis. Recursive Markov decision processes and recursive stochastic games. In Proc. of 32nd Int. Coll. on Automata, Languages, and Programming (ICALP'05), Springer, vol. 3580 of LNCS, 2005.

11. J. Filar and K. Vrieze. Competitive Markov Decision Processes. Springer, 1997.

12. M. R. Garey, R. L. Graham, and D. S. Johnson. Some NP-complete geometric problems. In 8th ACM STOC, pages 10-22, 1976.

13. M. Grötschel, L. Lovász, and A. Schrijver. Geometric Algorithms and Combinatorial Optimization. Springer-Verlag, 2nd edition, 1993.

14. T. E. Harris. The Theory of Branching Processes. Springer-Verlag, 1963.

15. R. J. Horn and C. R. Johnson. Matrix Analysis. Cambridge U. Press, 1985.

16. P. Jagers. Branching Processes with Biological Applications. Wiley, 1975.

17. K. Mahler. An inequality for the discriminant of a polynomial. Michigan Math J., 11:257-262, 1964.

18. D. A. Martin. Determinacy of Blackwell games. J. Symb. Logic, 63(4):1565-1581, 1998.

19. C. Manning and H. Schütze. Foundations of Statistical Natural Language Processing. MIT Press, 1999.

20. M. L. Puterman. Markov Decision Processes. Wiley, 1994.

21. U. G. Rothblum, P. Whittle. Growth optimality for branching Markov decision chains. Math. of Operations Research, 7: 582-601, 1982.

22. Y. Sakakibara, M. Brown, R Hughey, I.S. Mian, K. Sjolander, R. Underwood, and D. Haussler. Stochastic context-free grammars for tRNA modeling. Nucleic Acids Research, 22(23):5112-5120, 1994. 